\title{
XVIII.
}

\section{Bericht über die Thätigkeit meiner Klinik im Jahre 1896.}

\author{
Yon \\ Dr. Kretschmann, Magdeburg. ${ }^{\mathbf{1}}$ )
}

Der Zeitraum, welchen der vorliegende Bericht umfasst, reicht vom 1. Januar bis 31. December 1896. Es kamen währenddem als nea zugegangen notirt 936 Patienten in Behandlung. 123 von denselben erforderten stationäre Behandlung mit 970 Verpflegungstagen. Diesen reihen sich 11 Begleitpersonen mit 67 Verpflegungstagen an. Dem Alter und Geschlecht nach vertheilen sich die 936 Kranken wie folgt:

TABelle A.

\begin{tabular}{c|c|c|c}
\hline Lebensjahr & Männlich & Weiblich & Summe \\
\hline $0-10$ & 103 & 100 & 203 \\
$10-20$ & 104 & 88 & 192 \\
$20-30$ & 114 & 81 & 195 \\
$30-40$ & 110 & 64 & 174 \\
$40-50$ & 61 & 25 & 83 \\
$50-60$ & 33 & 20 & 53 \\
$60-70$ & 17 & 27 & 24 \\
uber 70 & 7 & 2 & 9 \\
\hline & 549 & 387 & 936 \\
\hline
\end{tabular}

Art und Verlauf der Krankheitsformen, deren 1304 an 936 Patienten beobachtet wurden, stellt folgende Tabelle dar:

1) Die früheren Jahresberichte sind im Selbstverlag erschienen. 
Tabelle B.

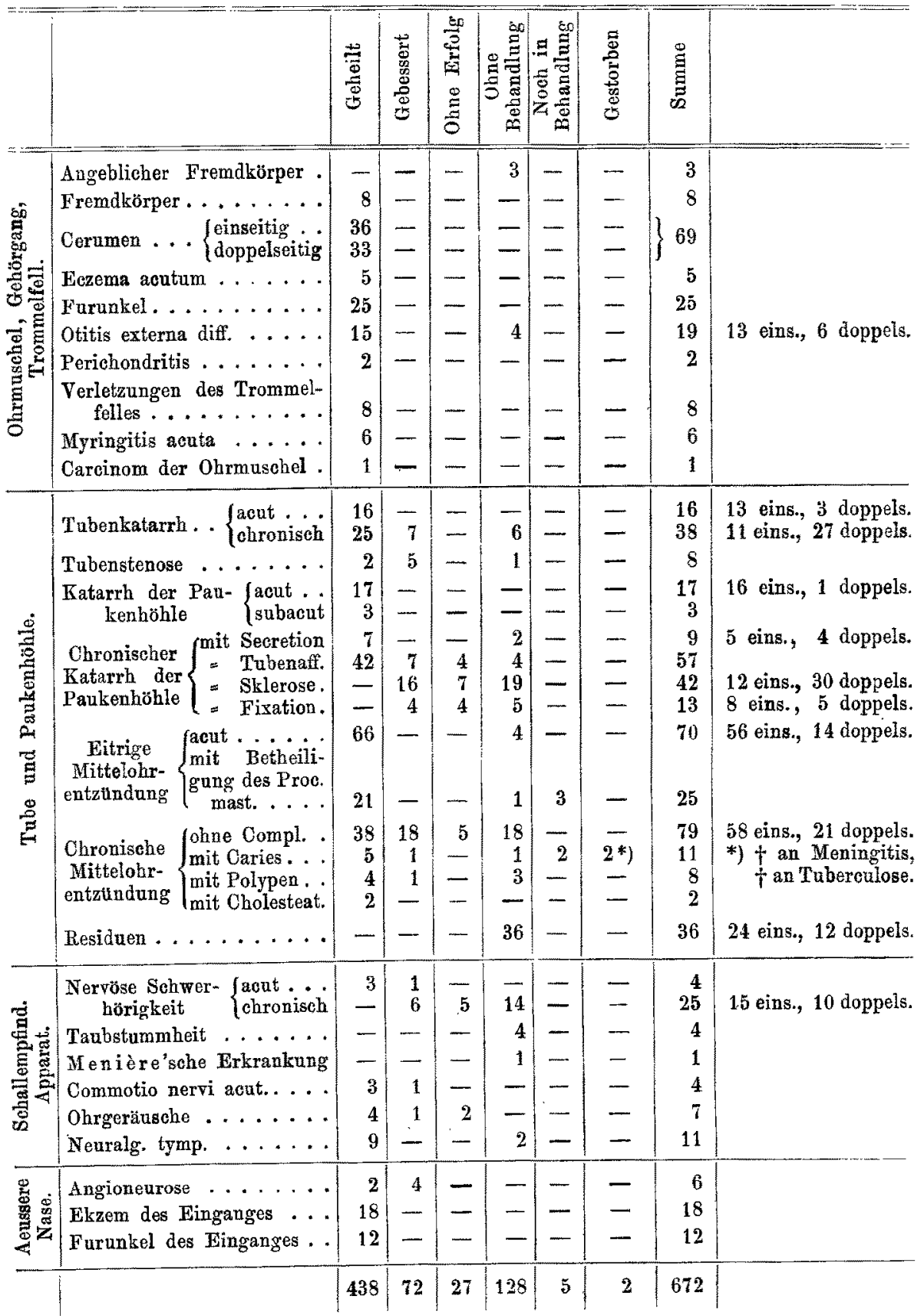


Bericht über die Thätigkeit meiner Kllinik im Jahre 1896.

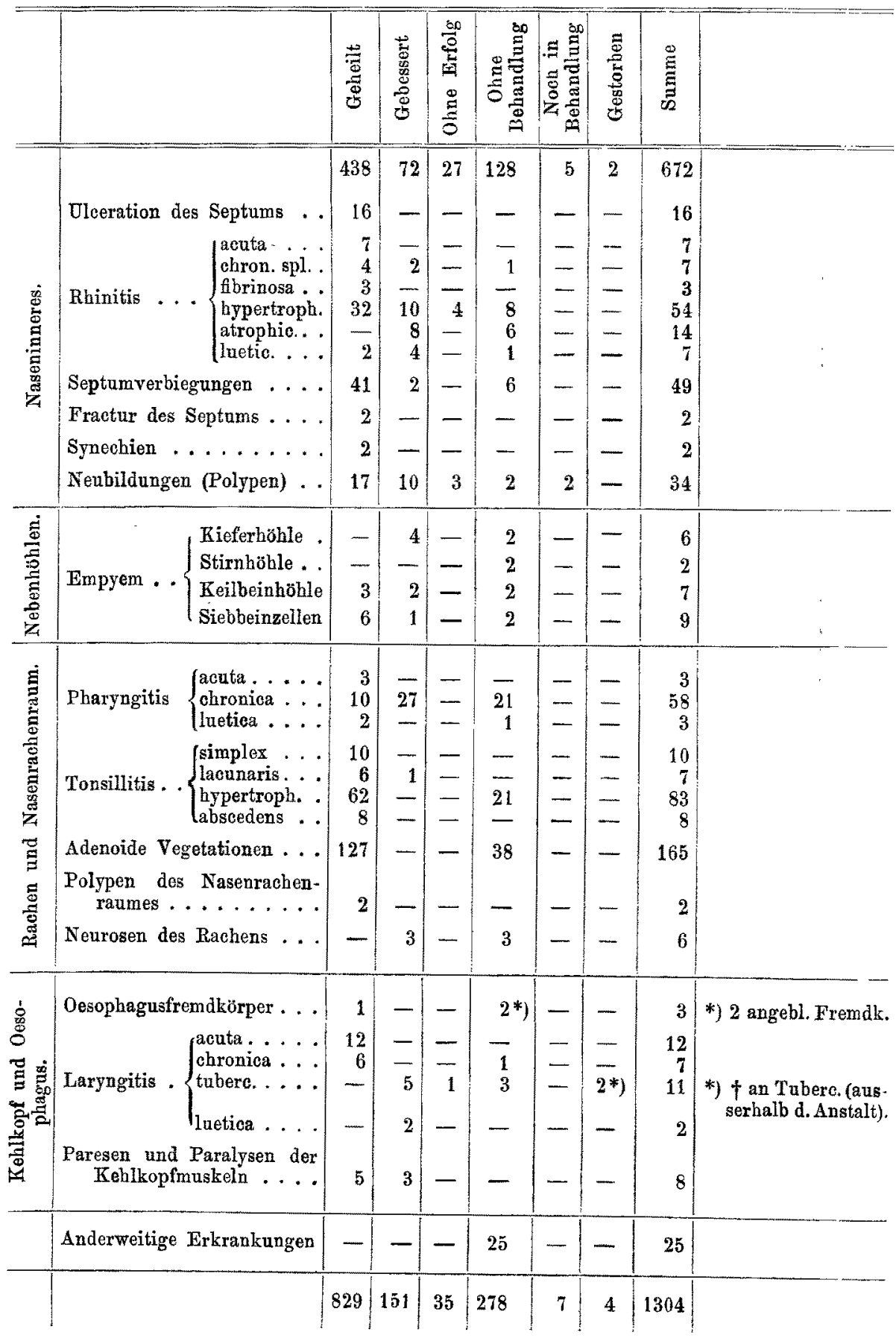


Die Anzahl der ausgefubrten. Operationen betrug 415 .

TABELLE C.

\begin{tabular}{|c|c|c|c|c|c|c|c|c|}
\hline \multirow[b]{2}{*}{ 今. } & & $\frac{\overrightarrow{7}}{9}$ & 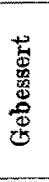 & $\begin{array}{l}\frac{80}{3} \\
\frac{2}{4} \\
0 \\
0 \\
0\end{array}$ & 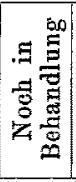 & 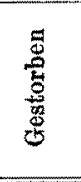 & 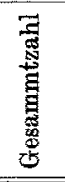 & \\
\hline & $\begin{array}{l}\text { Incision in den Gehörgang } \\
\text { Ineision bei Perichondritis } \\
\text { Polypenextraction ...... } \\
\text { Paracentese . . . . . . } \\
\text { Excision des Trommelfelles } \\
\text { Hammer-Amboss-Extraction } \\
\text { Ablösung der Musohel bei } \\
\text { Fremdkörpern . . . . } \\
\text { Trepanation des }\left\{\begin{array}{l}\text { acut } \ldots \\
\text { Chroniseh }\end{array}\right. \\
\text { Processus mastoid. }\end{array}$ & $\begin{array}{r}14 \\
2 \\
5 \\
25 \\
- \\
2 \\
\\
3 \\
19 \\
8\end{array}$ & $\begin{array}{r}- \\
-3 \\
-1 \\
1\end{array}$ & $\begin{array}{l}- \\
- \\
2 \\
4 \\
- \\
- \\
- \\
-\end{array}$ & $\begin{array}{l}- \\
- \\
- \\
- \\
- \\
- \\
3 \\
1\end{array}$ & $\begin{array}{l}- \\
- \\
- \\
- \\
- \\
- \\
\left.2^{*}\right)\end{array}$ & $\begin{array}{r}14 \\
2 \\
10 \\
29 \\
1 \\
3\end{array}$ & $\begin{array}{c}*) \text { an Meningitis, } \\
\dagger \text { an Tuberoulose. }\end{array}$ \\
\hline 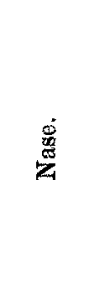 & $\begin{array}{l}\text { Entfernung von Polypen. } \\
\text { Entfernung } \text { v. Muschelenden } \\
\text { Synechientrennung . . . } \\
\text { Beseitigung } \\
\text { von Septum } \\
\text { deviationen }\left\{\begin{array}{l}\text { mit Trephine } \\
\text { mit Elektrolyse }\end{array}\right. \\
\text { Eröffnung der }\left\{\begin{array}{l}\text { Kieferhöhle } \\
\text { Keilbeinhöhle } \\
\text { Siebbeinzellen }\end{array}\right.\end{array}$ & $\begin{array}{r}17 \\
33 \\
2 \\
8 \\
33 \\
- \\
3 \\
6\end{array}$ & $\begin{array}{r}10 \\
10 \\
- \\
- \\
- \\
- \\
4 \\
2 \\
1\end{array}$ & $\begin{array}{l}- \\
- \\
-\end{array}$ & $\begin{array}{l}2 \\
- \\
- \\
- \\
- \\
-\end{array}$ & $\begin{array}{l}- \\
- \\
- \\
- \\
-\end{array}$ & $\begin{array}{r}29 \\
43 \\
2 \\
41 \\
\\
4 \\
5 \\
7\end{array}$ & \\
\hline \multirow[t]{3}{*}{ 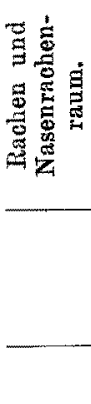 } & $\begin{array}{l}\text { Abtragung }\left\{\begin{array}{l}\text { einseitig . . } \\
\text { der Tonsillen } \\
\text { doppelsetig }\end{array}\right. \\
\text { Entfernung adenoider Vege- } \\
\text { tationen } \ldots \ldots \\
\text { Nasenrachenpolypen . . . . }\end{array}$ & $\begin{array}{r}5 \\
57 \\
127 \\
2\end{array}$ & - & - & $\begin{array}{l}- \\
- \\
-\end{array}$ & $\overline{-}$ & $\begin{array}{r}127 \\
2\end{array}$ & \\
\hline & $\begin{array}{l}\text { Jugulaxisunterbindung und } \\
\text { Trepanation auf mittlere } \\
\text { u. hintere Schädelgrube }\end{array}$ & - & - & - & - & 1*) & 1 & $\begin{array}{l}\text { *) Vgl. } † \text { an Menin- } \\
\text { gitis unter Trepa- } \\
\text { nation des Pruc. } \\
\text { mast. }\end{array}$ \\
\hline & & 374 & 26 & 6 & 6 & $2(+1)$ & 415 & \\
\hline
\end{tabular}

Hieran anschliessend mögen einige Krankengeschichten Platz finden, die eines gewissen Interesses nicht entbehren.

Acute Mittelohrentzündung, Sinus- und Jugularisthrombose, Tod an Meningitis purulenta.

Christ of M., 60 Jahre alt, lange Jahre in Maschinenwerkstätten beschäftigt, bekam wenige Tage vor Weihnachten 1895 beftige Schmerzen im linken Ohr. 4 Tage hatten dieselben bestanden, als er sich am 26. Decbr. vorstellte. Das linke Trommelfell fand sich in seiner oberen Circumferenz 
vorgewölbt und geröthet. Die unteren zwei Drittel waren von normaler Färbung, Lichtreflex vorhanden, Hammer stark retrahirt. Es handelte sich auscheinend um eine Entzündung des oberen Paukenhöhlenabschnittes, der durch Verwachsung des Trommelfelles mit der Labyrinthwand von der übrigen Paukenhöhle abgeschlossen war. Rechterseits Trommelfell stark retrahirt, Gehör gleich 0. Weber nach links lateralisirt, links Conversationssprache dicht am $\mathrm{Ohr}$. Knochenleitung fehlt. Stimmgabel von $\mathrm{C}$ bis $\mathrm{c}^{4}$ wahrgenommen.

Paracentese entlang dem oberen Rand des Margo tympanicas entleert Eiter. Schmerzen lassen nach. Da er uber Frösteln und dumpfes Gefuhl im Kopf klagt, erfolgt am 29. Aufnahme in die Anstalt. Temp. Abends $39,2^{\circ}$. Puls 92, Frostgefühl. Am 30. Paracentese wiederholt. Die Temperaturen der nächsten Tage überschreiten $38,2^{\circ}$ nicht. Kopfschmerz besteht fort. leichtes Ziehen im Nacken. Am 3. Januar Schmerzhaftigkeit des linken Schultergelenkes, kein nachweisbarer Erguss. Temperatur $38,8^{\circ}$. Trommelfell blass, Paracentesenöffnung sichtbar, keine Secretion. Spitze und hinterer Rand des Processus druckempindlich, geringes Oedem in der Warzenfortsatzgegend. Die Temperaturen in den nächsten Tagen sind normal, einige Vlale tritt in den Nachmittagsstunden Schweisseruption, ohne Frostgefühl oder Temperatursteigerung auf. Am 10. wird die Paracentese wiederholt. Die örtlichen Erscheinungen in der Warzengegend gehen zurück und kehren wieder. Die Untersuchung des Augenhintergrundes ist nicht möglich, da rechts Hornhautnarben den Einblick hindern, links das Auge vor Jahren entfernt worden ist.

Am 19. Aufmeisselung des Warzenfortsatzes. Corticalis gesund. Das Innere des Knochens von Granulationsmassen erfüllt, in diese mehrere Sequester eingebettet. Durchbruch an der medialen Wand, daher Fortnahme der ganzen Spitze. Im Antrum selbst kein Eiter. Ein Durchbruch in die mittlere oder hintere Schädelgrube ist nicht vorhanden.

Nach dem Eingriff wesentliche Besserung des Befindens, Temperatur und Puls normal, geringer Kopfschmerz besteht noch.

23. Januar Verbandwechsel, Wunde sieht gut aus, Oedem zurückgegangen. Pulsfreguenz 104 Schläge, Temperatur normal.

Am 24. Januar. Gegen Mittag paroxysmal auftretender Kopfschmerz, der in den Scheitel verlegt wird, in der folgenden Nacht grosse Unruhe, Trübung des Sensoriums, Temperatur steigt aut $40^{\circ}$, Puls 128.

25. Januar früh. Freilegung des Sinus transversus. Wandung gesund, Resistenz für den palpirenden Finger. Nach Incision der Wand findet sich ein rother obturirender Thrombus. Bei der Verfolgung nach dem Bulbus zu quillt Eiter unter Pulsation hervor. Nach der Peripherie hin ist der Thrombus gesund, deshalb wird die Freilegnng nicht weiter als $2 \mathrm{Cm}$. oberhalb der Umbiegungsstelle fortgesetzt, die Thrombusmassen ausgeräumt. Jugularis an der Grenze des unteren und mittleren Drittels im Gesunden unterbunden. Im oberen Viertel eitrig zerfallener Thrombus. Excision dieses ganzen Stückes. Die Punction des linken.Schläfenlappens und der linken Kleinhimhemisphäre, welche nach Freilegung dieser Theile vorgenommen wurde, fiel negativ aus. Der Eingriff fürte keine Besserung herbei. Die Unruhe blieb bestehen, um gegen Abend in tiefe Somnolenz aberzugehen. Am 27 . fruh erfolgte der Exitus im Koma. Schädelsection 3 Stunden post mortem:

Schädeldach sebr dick, schwer von der Dara abziehbar. Dura über den Stirnlappen blasig abgehoben und geknittert, im Uebrigen nicht sehr gespannt. Im Sinus longitudinalis und rechtem Transversus füssiges Blut. Im linken Transversus findet sich ein Thrombus ca. $2 \mathrm{Cm}$. vom Torcular beginnend und bis zu dem durch die Operation geschaffenen Schnitt reichend von ca. $2 \mathrm{Cm}$. Länge, dunkelrother Farbe, im Inneren eitrig erweicht. Der Sin. petros. sup. u. inf. ebenso wie der Cavern, sind gesund. Nach Entfernung der Dura findet sich ein mächtiges, eitriges Kxsudat gleichmässig auf der Convexität beider Hemisphären, das die Form der Gyri völlig verdeckt und beim Abziehen der Pia reichlich aus den Sulcis abtropft. Geringere, wenn auch noch sehr bedeutende Exsudatmassen finden sich an den einander ztigewandten medialen Flächen der Hemisphären und in den Fossae Sylvii. An der Basis des Gehirnes ist keine Spur von Exsudat zu finden, ebensowenig am ganzen Kleinhirn mit Ausnahme einer geringen Auflagerung auf der oberen Fläche des 
Oberwurmes. Am Schläfenlappen und Kleinhirn finden sich einige blutig tingirte Stellen, hervorgerufen durch die Punctionen bei der Operation.

In den Ventrikeln keine Flüssigkeit. Die Hirnsubstanz nicht ödematôs. Abscesse fehlen. Nach Entfernung der Dura von der Schädelbasis zeigt sich die letztere frei von pathologischen Veränderungen.

Die linke Felsenbeinpyramide wurde zu genauerer Untersuchung der Leiche entnommen. Im Bulbus venae jugularis dickflüssiger Eiter. Die Intima getrübt, verdickt und gefaltet. Der Knochen der Knppel für den Bulbus v. jug. erweist sich gesund. Entfernung der unteren knöchernen Gehörgangswand. Trommelfell stark retrahirt, Paracentesenöffnung geschlossen. Bei der Entfernung des Tegmen tympani muss eine in sagittaler Ebene verlanfende Membran (Lig. mallei et incudis sup.) von der Knochenplatte getrenut werden. Diese Membran von grosser Mächtigkeit beginnt an der vorderen PaukenFand, um in der Ebene des Hammerkopfes und Ambosshörpers verlaufend am lateralen Pfeiler der Eingangsöffnung ins Antrum zu inseriren. Es erscheint das Köpfchen des Hammers und ein Stück des Ambosskörpers. Alle anderen Gebilde, sowie der tiefere Einblick in die Paukenhöhle werden verhüllt durch eine diaphragmaartige, horizontal verlaufende Membran, die vom oberen Rand des tympanalen Tubenostium zur unteren Grenze der Zugangsöffnung zum Antrum zieht in der Weise, dass der $\mathrm{R}$ ivin i'sche Ausschnitt und ein kleines Segment des Margo tympanic. sup. et post, in den durch jene Membran gebildeten oberen $\mathbf{P a u k e n h o ̈ h l e n r a u m ~ m i t ~ e i u b e z o g e n ~ i s t . ~}$

Jetzt wird das Trommelfell in seiner unteren Circumferenz nmschnitten, der Boden der Pankenhöhle entfernt. Es zeigt sich eine feste Verwachsung zwischen Umbo und Promontorium. Oberhalb dieser Verwachsungsstelle bis zu jener Diaphragma ähnlichen Membran hin ist der ganze Raum der Pankenhöhle erfült von einom mächtigen Bindegewebspolster, das nur mit Mühe eive Trennung des Trommelfelles von der Labyrinthwand ermöglicht. Dieses Bindegewebspolster fúlt das tympanale Ostium der Tuba, die Nische des runden Fensters und des ovalen vollständig aus. Nur mit Mühe ist der Stapes aus diesen Bindegewebsmassen zu entwickeln. Seine Fussplatte ist sonst gut beweglich.

Der vorliegende Fall ist in mehrfacher Hinsicht nicht obne Interesse, einmal wegen des klinischen Verlaufes, ferner wegen des eigenartigen Sitzes der Meningitis und drittens wegen der Veränderungen im Mittelohr.

Die zweite Operation und die Obduction haben gezeigt, dass wir es im vorliegenden Falle mit einer ansgedehnten Sinusthrombose zu thun hatten. Leider kam die Ausräumung des Thrombus und die Unterbindung der Jugularis zu spät, da bereits die tödtliche Meningitis eingesetzt hatte. Nun waren ja manche Symptome vorhanden, die auf Phlebitis des Sinus hinwiesen, so der im Beginn der Erkrankung aufgetretene Frost mit Temperatursteigerung höheren Grades, die Schmerzhaftigkeit des linken Schultergelenkes, die wechselnden Erscheinungen in der Warzenfortsatzgegend, aber das sind doch alles Erscheinungen, die sich finden können auch bei Abwesenheit eines grösseren Thrombus, die jedenfalls nicht mit Sicherheit für einen solchen sprechen. Das markanteste Symptom, was wir sonst für Sinusthrombose kennen, die wiederkehrenden Schuittelfröste, die steilansteigenden und ebenso abfallenden Temperatureurven, fehlte. Die Temperaturen waren ab- 
gesehen vom Beginn und vom Ende meist normal, zuweilen wenige Zehntel die Norm uberschreitend. Zu alle dem kam der günstige Effect, welchen die Aufmeisselung und Ausräumung des Warzenfortsatzes hervorrief.' Da keine Veränderungen am Knochen sich fanden, so standen wir von einer Freilegung des Sinus ab. Dass wir diese Freilegung unterlassen haben, war ein Missgriff, wie dieser Fall überzeugend darthut. Wenn man bedenkt, wie gering die Gefahr ist, welche aus der Exploration des Sinus erwachsen kann im Verhältniss zu dem grossen Gewinn, den eine rechtzeitige Entdeckung des Thrombus und dessen Entfernung für den Träger bringt, so sollte man in Fällen, die auch nur die Möglichkeit einer Sinusphlebitis andeuten, nicht zögern mit der Freilegung jenes Blutleiters. Unser Fall zeigt wieder einmal, dass, will man bei der Feststellung intracranieller Complicationen erst unzweideutige Zeichen abwarten, die Hülfe vielfach zu spät kommt. Es ist besser, lieber einmal lediglich diagnostisch eingegriffen $\mathrm{zu}$ haben, als hinterher zu der Erkenntniss zu kommen, dass durch Zaudern der unglitckliche Ausgang verschuldet ist.

Bei dem Sectionsbefund erregt besonderes Interesse die Localisation des meningitischen Exsudates. Dasselbe fand sich ausgebreitet auf der Convexität der Grosshirnhemisphären, an ihren medialen Flächen und lateralwärts in den Sylvi'schen Gruben. Wir haben es hier also mit einer reinen Convexitätsmeningitis zu thun, einer Form, welche nach Körner ${ }^{1}$ ) sehr selten zu sein pflegt.

Ein manifester Zusammenbang mit dem primären Eiterherd, der doch sicherlich von dem zerfallenden Thrombus gebildet wird, ist nicht nachzuweisen. Die Paukenböhle ist wohl kaum als verursachender Eiterherd in Anspruch zu nehmen, da die in ihr ursprïnglich etablirte Entzündung zur Zeit der Entstehung der Meningitis bereits abgelaufen war. In Anbetracht dieses Befundes gewinnt die von Körner (l. c.) ausgesprochene Ansicht, dass die Meningitis in Fällen, ähnlich dem vorliegenden, nicht als direct übergeleitete, sondern als metastatische aufzufassen sei, erheblich an Wahrscbeinlichkeit. Von Interesse waren auch die Verhältnisse in der Paukenhöhle. Nur der obere und untere Abschnitt stellten noch einen Hohlraum dar, der ganze Mitteltheil war durch Bindegewebe obliterirt und nicht lufthaltig. Der obere Raum war durch eine von der Höhe des Hammerkopfes und Ambosskörpers

1) Die otitischen Erkrankungen des Hirnes u. s. w. 2. Auf. S. 42. 
nach dem Dach der Paukenhöhle ziehende sagittale Membran in zwei Theile geschieden, ron denen der medial gelegene allein mit dem Antrum in Verbindung stand. Der laterale war vollständig in sich abgeschlossen und zeigte nirgends Communication mit Nebenräumen. Ein derartiger Abschluss des HammerambossSchuppenraumes hat bei der Anordnung der Bänder, welche von den Gehörknöchelchen zu den Wänden der Paukenhöhle ziehen ${ }^{1}$ ) nichts Wunderbares und kommt ja auch in der That nicht selten zu Stande. Ob er: auf das Zustandekommen von intracr. Complicationen von Einfluss ist, das mag dahingestellt bleiben.

An dem Thrombus konnten wir zwei Zerfallsherde constatiren. Der eine grössere umfasste das letzte Stück des Sinus vor seiner Einmündung in den Bulbus, diesen selbst und das Anfangsstlick der Jugularis. Der andere Herd fand sich im peripheren Ende des Thrombus. Es sind dies gerade Stellen, die räumlich ziemlich weit von dem oberen Paukenraum, dem primären Sitz der Eiterung, entfernt liegen. Für die Infection des unteren Thrombusabschnittes giebt ja das Uebergreifen der Eiternng auf die Warzenzellen, besonders die in der Spitze gelegenen die nöthige Erklärung, für den peripheren Zerfallsherd liegt eine solehe Erklärung nicht vor, und es drängt sich deshalb die Frage auf, ob dieselbe nicht als eine Metastase aufufassen ist, eine Frage, die im vorliegenden Fall insofern nicht ohne Berechtigung ist, als wir ja auch bei der Meningitis die metastatische Entstehung für wahrscheinlich hielten.

Acute eitrige Mittelohrentzündung, Meningitis einsetzend, nachdem die Paukenerkrankung nabezu abgelaufen war.

Herr K., 44 Jabre, Kaufmann, bekam nach einem schnupfen rechterseits heftige Ohrschmerzen. Tags darauf am 18. Novbr. 1895 konnte eine Röthung and Abflachung des Trommelfelles constatirt werden. Als nach Burrowschen Umschlägen die Schmerzen anhalten, Paracentese am 20, November. Darauf treten die Schmerzen nur vorübergehend anf und sind von kurzer Daner. Die Anfangs seröse Secretion wird nach vier Tagen sehr reichlich dickeitrig. Die folgenden drei Wochen keine wesentliche Aenderung. Jetzt tritt Oedem am Warzenfortsatze auf ohne nenvenswerthen Druckschmerz. Der Vorschlag einer Operation wird abgelehnt. Bei energischer Eisbehandlung geht das Oedem zuräck, die Secretion nimmt $a b$, und Ende December ist die Perforation geschlossen, ' das Trommelfell blass, Hammergriff sichtbar. Gehör für Flüster. sprache 2\%: Mtr. Am 5. Januar 1896 Paukenhöhle frei. Schmerzen sind verschwunden, es besteht noch eine Neigung zu leichtem Schwindel. Am 12. Januar Morgens wurde ich in die Wohnung des Kranken gerufen. Da beim Versuch, aufzustehen, starker Schwindel, Uebelkeit und Brecbneigung aufgetreten war. Fieber war nicht vorhanden, Pals 76 nicht gespannt, keine

1) Vergl. darüber meine Arbeit: Fistelöffnungen am oberren $P_{0} l e$ des Trommelfelles. Dieses Archiv. Bd. XXV. S. $169 \mathrm{ff}$. 
Schmerzen, keine Veränderung am Warzenfortsatz. Trommelfell blass, von normaler Wölbung. Weber nach links lateralisirt, rechts Taubheit gegen Flüsterworte und Stimmgabeln Knochenleitung aufgehoben.

Aufnahme in die Klinik. Paracentese fördert kein Secret zu Tage, doch hat man das Gefühl, als wenn sich die Nadel nach Durchtrennung des Trommelfelles in einem dicken Schleimhautpolster befände.

Am 13. Paracentesenwunde reactionslos, kein Secret, Puls und Temp. normal, Kopfschmerz in der Stirngegend. Schwindel unbedentend.

14. Januar. Geringer Schleimp fropf im Gehörgang, kein Schwindel, keine Temperaturstörung, Patient verlässt auf eine Stunde das Bett.

15. Januar. In der verflossenen Nacht wäthende Stirnkopfschmerzen, Morgentemperatur $38^{\circ}$, Puls 84 , Mittags $39,4^{\circ}$. Puls 110 , Augenhintergrund normal, Sensorium nicht mehr frei. Abends $39,8^{\circ}$, Puls nicht zu zâhlen, Somnolenz.

16. Januar. Die Nacht hat Pat. viel gestöhnt, lässt Stuhl unter sich, Pupillendifferenz. Tod um 9/2 Uhr.

Kopfsection 6 Stunden post mortem.

Schädeldach von normaler Dicke, Diploë ziemlich blutreich. Dura stark gespannt. In den grossen Blutleitern keine pathologischen Veränderungen. Piavenen reichlich gefült. Die Grosshimbemisphären von einem truben, sulzigen Exsudat überlagert, rechts in grösserer Ausdehnung wie links. An der Basis ist die Entwicklung des Exsudates auf der Oberfläche und in der nächsten Umgebung des Pons am bedeutendsten, geringer beiderseits in den Sylvi 'schen Gruben.

In den Ventrikeln reichliche Flüssigkeitsansammluag von milchiger Farbe. In der Hirnsubstanz keide Veränderung, ebensowenig an der basalen Dura. Nach Entfernung der letzteren zeigt sich an der rechten Pyramide entsprechend der Convexität des oberen Bogenganges der Knochen in Linsengrösse röthlich verfärbt, erweicht, aber nicht eingeschmolzen.

Das rechte Trommelfell ist verdickt, Paracentesenöffnung im vorderen, unteren Quadranten. Die Schleimhaut der Paukenböhle und des Antrums stark geschwollen, so dass ein Lumen nicht mehr existirt, die Gehörknöchelchen intact. Die Membran des runden Fensters verdickt, die Fussplatte des Steigbügels und ihr Befestigungsband nicht verändert. An der Labyrinthwand dort, wo der horizontale Bogengang dieselbe erreicht, eine stecknadelkopfgrosse, röthliche Verfärbung und Erweichung des Knochens. Der Vorhof erfüllt von einer röthlichen pulpösen, zähen Masse, ebenso wie die Schnecke und die Bogengänge.

Im Por. acustic. int. kein Exsudat, desgleichen nicht in der Scheide der in ihm verlaufenden Nerven. Die Bohlräume des Warzenfortsatzes sind luftleer, erfüllt von einem homogenen mucösen Gewebe ohne Flüssigkeit.

Ungewöhnlich an diesem Fall ist das Auftreten einer Leptomeningitis zu einer Zeit, wo man den entzündlichen Process in der Paukenhöhle als nahezu abgelaufen bezeichnen durfte. Leider haben wir es unterlassen, Culturen anzulegen, und uns so der Möglichkeit begeben, vielleicht dieses nicht gewöhnliche Krankheitsbild za klären. So sind wir eben nur auf die Vermuthung angewiesen, dass es sich um eine Mischinfection gehandelt haben könnte von Entzündungserregern, deren Entwicklung und Entfaltung der. Virulenz zeitlich wesentlich different gewesen ist. Der Weg der Eiterung lässt sich bis an die Oberfläche der Pyramide leicht verfolgen. Dort verliert sich die Spur. An der basalen Dura, an den aus dem Porus. acust. intern. austretenden Stämmen finden wir makroskopisch keine Veränderungen, die auf die Fährte 
der Verbreitung der Eiterung führten. Wenn wir aber annehmen dürfen, dass die Stelle der bedeutendsten Entwicklung des Exsudates die Gegend des Pons, auch die der Zeit nach zuerst ergriffene gewesen ist, so dürfen wir doch vielleicht auf die Bahnen des 7 . und 8. Nerven als Leiters der Eiterung zurückgreifen trotz fehlenden directen Nachweises, und uns den Zusammenhang so erklären; dass bei dem schnellen Verlauf der Erkrankung eine nachweisbare Exsudatentwicklang entlang der Nervenstämme nicht zu Stande gekommen ist.

Anderenfalls miissen wir uns mit der Thatsache begnügen, dass wie im vorigen Falle die Hirnhautentzlindung sich entwickeln kann, obne dass ein nachweisbarer directer Zusammenhang mit dem primären Eiterherd besteht.

\section{Arrosion der Sinuswand durch Cholesteatom.}

Frau E., 68 Jahre alt, leidet seit 15 Jahren an eitrigem Ausfluss des rechten Ohres. Während sie in der ersten Zeit gar keine Beschwerden gefählt hat, leidet sie in den letzten 3 Jahren häufig an Schwindel, der ganz besonders heftig auftritt, wenn sie mit einer Haarnadel ins $\mathrm{Ohr}$ kommt. Bei der Untersuchung fand sich die hintere knöcherne Gehörgangswand usurirt, der Warzenfortsatz und die Paukenhöhle von Cholesteatommassen erfüllt. Bei ganz leiser Berührung mit der Sonde wurde ein heftiger Schwindelanfall ausgelöst, so dass die Kranke gehalten werden musste, um nicht vom Stuhl zu stürzen. Bei der Operation fand sich der ganze Warzenfortsatz excavirt und mit Cholesteatomschollen ausgestopft. Da bei der grossen Neigung zu Schwindel an einen Durchbruch ins Labyrinth gedacht werden musste, so wurden die Massen mit grosser Vorsicht entfernt. Sie liessen sich leicht in toto heraus heben. Im Moment der Entfernung trat eine starke venöse Blutung auf. die nur dem Sinus entstammen konnte. Durch 'Tamponade kam sie sehr bald zum Stehen. Es zeigte sich nun der Querblutleiter in Ausdehnung von $11 / \mathrm{Cm}$. freiliegend. Seine Wandung war glatt, nirgends missfarbig und schimmerte blau durch, Auf der Mitte des freiliegenden Stückes lag eine Cholesteatomscholle von ungefähr Linsengrösse. Sobald dieselbe mit dem Tupfer weggewischt war, trat erneute Blutung anf, die nach abermaliger Tamponade stand. Jetzt konnte man an der Stelle, an der die Scholle gelegen hatte, eine kleine, zapfenförmige Vorwölbung constatiren, auf deren Spitze eine stecknadelkopfgrosse Fistel sich befand. Dabei war auch hier von einer Verfärbung keine Spur zu tinden. Eine Fistel nach dem Labyrinth war nicht vorhanden. Die Heilung verlief glatt und fieberlos. Die Schwindelanfälle kehrten nicht wieder.

Wie ist nun die Continuitätstrennung in der Sinuswand zu Stande gekommen? Eine Verletzung bei der Operation ist ausgeschlossen. Es war nur eine ganz dünne Knochenplatte abzudecken, um die Geschwulst gänzlich freizulegen. Es wurde ferner sehr vorsichtig die Masse stumpf herausgehebelt. Erst beim Lüften des Tumors trat die Blutung auf, weil, wie sich aus dem nachherigen Befund ergab, bei der Entfernung die als Ventil dienende Cholesteatomlamelle mit gehoben sein wird. Von innen nach aussen wird auch schwerlich der Durchbruch erfolgt sein. Es 
hätten ja in diesem Falle im Inneren des Gefässes ulcerirende Processe stattfinden müssen, und diese würden kaum symptomlos verlaufen sein. Es ist aber weder vor, noch nach der Operation Fieber vorhanden gewesen. Somit bleibt nur die Möglichkeit, dass der Durchbruch von aussen nach innen erfolgt ist. Veränderungen entzündlicher Natur liessen sich an der Gefässwand nicht nachweisen. Wir dürfen deshalb wohl annehmen, dass der mechanische Druck des Cholesteatoms die Usur veranlasst hat. Die Form der zitzenförmigen Prominenz spricht dafür, dass die Continuitätstrennung zuerst an der dem Gefässlumen abgewandten Seite der Wandung erfolgt ist, dass der Blutdruck dann die Intima und ev. Media gleichsam herniös vorgetrieben hat, und dass erst in einem späteren Stadium der Durchbruch auch dieser Membranen erfolgt ist. Der Verlauf der Operation macht es wabrscheinlich, dass nachträglich im Gefässinneren schwerere Veränderungen nicht stattgefunden haben, beweist also die besonders von Leutert ${ }^{1}$ ) vertretene Ansicht, dass Eröffnungen des Sinus ohne Bedeutung sind, wenn ein fortdauernder Zufluss septischen Materials nicht stattfindet. Andererseits würde, wenn die Operation nicht gerade noch rechtzeitig eingegriffen bätte, eine consecutive Sinusthrombose mit Sicherheit zu erwarten gewesen sein.

\section{Zwei Fälle von Perichondritis des Gehörganges.}

Wil.h elm $R$., 52 Jahre alt, bekam 14 Tage vor seiner Aufnahme heftige, rechtsseitige Ohrschmerzen. Er behandelte dieselben mit Kamillenumschlägen, ohme Nachlass zu ervielen. Bei der Untersuchung zeigte sich im Anfangstheil der unteren Gehörgangswand eine Vorwölbang, die ganz den Eindruck eines grossen Furunkels machte. Auf der Höhe fand sich die Hant durchbrochen, und es entleerte sich Eiter aus der Oeffnung. Da der Abfluss nicht genügend schien, wurde eine ausgiebige Incision gemacht. Das Gefühl, was man bei der Incision hatte, war eigenthümlich krepitirend. Der Schnitt wurde mit Jodoformgaze tamponirt und täglich frisch verbunden. Es zeigte sich indessen keine Tendenz zur Heilung, vielmehr trat im unteren Drittel der Ansatzlinie der Ohrmuschel eine leichte Anschwellung anf. Da die Sondirung den freiliegenden Knorpel nachwies, wurde in Narkose von der Gehörgangswunde aus mit dem scharfen Löffel eingegangen und eine Anzahl Knorpeltrümmer von Hirse- bis Reiskorngrösse entfernt. Dabei wenig Eiter. In dem unteren Drittel der Ansatzlinie der Ohrmuschel wurde eine Gegenöffnung angelegt und ein Drainrohr eingelegt, dessen eines Ende aus diesem Schnitt, das andere aus der Gehörgangsöffnung hervorragte. Die Heilung verlief nun ganz glatt. In vierzehn Tagen waren die Schnitte geschlossen, eine Deformităt des Gehörganges blieb nicht zurück.

Ganz ähnlich ist der zweite Fall.

Frau F., $49 \mathrm{~J}$, war an linksseitigen Ohrschmerzen erkrankt, der Hausarzt hatte einen Furunkel constatirt und denselben breit incidirt. 2 Wochen nach Beginn der Erkrankang sah ich die Patientin. Es fand sich an der

1) Dieses Archiv. Bd. XLI. S. 217 ff. 
unteren Gehörgangswand ein klaffender Schnitt, aus dem rabmiger Fiter quoll. Nach Reinigung der Wunde sah man auf dem Grande eine graugelbliche Masse, die bei Sondenberührung als blossliegender Knorpel sich erwies. Eingedenk des vorherigen Falles machten wir hier ebenfalls an gleicher Stelle eine Gegenöffnung und schabten den Knorpel mit scharfem Löffel aus. Es zeigte sich nun ein gelöstes Stück von ca. $1 \mathrm{Cm}$. Durchmesser. Eitermenge gering. Drainage ebenso wie im vorigen Fall. Heilung in 14 Tagen. Der Gehorgang erscheint an seiner unteren Wand muldenförmig vertieft.

In beiden Fällen ist der Entwicklungsgang der gleiche. Eine anseheinend circumscripte Otitis externa, vielleicht im Anfang unzweckmässig selbst behandelt, hat auf das Perichondrium übergegriffen, dasselbe konnte seine den Knorpel ernährende Function nicht mehr ausüben, und es kam zur Nekrose, die im zweiten Falle erheblich ausgedehnter war, als im ersten. Die Entfernung der nekrotisirten Theile fubrte schnell zur Heilung. Zucker und Eiweiss war in dem Urin beider Patienten nicht vorhanden.

Es kam im Verlaufe des Berichtsjahres noch ein dritter Fall von Perichondritis zur Behandlung. Derselbe verlief aber weit ungünstiger. A usgedehnte Pblegmonen in der Umgebnng schlossen sich an, und es kam zu weitgreifender Nekrose des Unterkiefers. Da der Fall noch nicht abgelaufen ist, soll erst bei einer späteren Gelegenheit darïber eingehend beriehtet werden.

\section{Hysterie unter dem Bilde von Taubbeit.}

Luise N., 9 Jahre, hat nach Angabe des bebandelnden Arztes 8 Wochen vor der Aufnahme einen Typhus durchgemacht, in dessen Gefolge sich beiderseitiger Vittelohrkatarrh entwickelte, der zu völliger Taubheit führte. In den letzten zwei Wochen sollen wieder vereinzelte Hörempfindungen vorhanden gewesen sein. Die Mutter des Kindes giebt an, dass dasselbe zu seinen Angehörigen mehrfach geäussert habe: "1hr müsst langsam sprechen, ich höre Euch wohl, kann Euch aber nicht gleich verstehen." Das Kind, etwas mager, macht keinen kranken Eindruck. Objectiv finden sich an den Gebörorganen keine Abnormitäten. Das rechte Kniegelenk ist ankylotisch infolge einer vor mehreren Jahren ausgefubrten Resection. Der Gang ist eigenthümlich. Das Kind läuft auf den Fussspitzen, hält den Körper leicht vorgebeugt, setzt die Hacken nicht auf. Die Bewegangen sind steif und ungelenk. Hin Schwanken oder Taumeln selbst bei geschlossenen Augen findet sich nicht. Die Reflexe sind normal. Die elektrische Erregbarkeit der Muskeln weist auf keine pathologische Veränderung. Auf laut vorgesprochene Sätze oder Worte reagirt sie nicht, dagegen scheinen die Töne im Bereich von $\mathrm{C}$ bis $\mathrm{c}^{3}$ wahrgenommen zu werden, sowohl bei Luftleitung, wie durch den Knochen. In den ersten 6 Tagen wurden Pilocarpininjectionen angewendet, ohne jeden Erfolg. Da nach den Angaben der Mutter auf vorhandene Hörreste geschlossen werden konnte, so wurde versucht, durch Hörubungen das Verständniss für Sprachlaute wieder zu wecken. Vocale wurden im Ganzen richtig, wenn auch eimigermaassen zögernd nachgesprochen. "i“ und "̈̈ "schienen nicht wahrgenommen zu werden, statt "eu" wurde "ei", statt "au" "o" geantwortet. Sehr bald stellte es sich heraus, dass dieses Resultat durchaus inconstant war. Dieselbe Bemerkung machten wir auch bei Worten, mochten sie ein- oder mehrsilbig sein. Um nun ganz sicher zu gehen, wurden in jeder Sitzung die Worte, welche sie wiederholt richtig nachsprach, notirt, ebenso wurde bei den unrichtig nach- 
gesprochenen auch die entstellte Wortform mit aufgezeichnet. Wurden nun in späteren Sitzungen die so aufgezeichneten Ausdrücke wieder examinirt, so geschah es häufig, dass die Anfangs richtig nachgesprochenen Worte falsch, die falschen richtig wiedergegeben wurden. Gelegentlich einer Untersuchung der Abdominalorgane, bei welchem Act das Kind grosse Angst zeigte, fiel es uns auf, dass sie allen an sie gerichteten Befehlen pünktlich nachkam, anch dann, wenu sie das Gesicht des Sprechenden nicht sehen konnte. Allen Zweifel aber, dass es sich um Taubheit physischer oder psychischer Art handeln könute, benahm die Bemerkung, dass das Kind im Umgang mit anderen Kindern, die längere Zeit mit ihm zusammengelegen hatten, durchaus keine Hörstörungen zejgte. Die Unterhaltung ging so flott von Statten, wie bei einem hörgesunden Kinde. Allerdings war das nur der Fall, wenn sie sich unbeobachtet glaubte. Wurde sie eines Erwachsenen, mochte er vom Personal oder Mitkranken sein, ansichtig, dann wurde sie wortkarg und reagirte nur höchst unvollständig auf an sie gerichtete Worte. Bei der Behandlung kam es darauf an, dem Kinde thunlichst klar zu machen, dass sein angeblicher Hördefect zu Unrecht bestände. Es wurden ihr kurze Sätze dictirt, welche es nachzuschreiben und dann vorzulesen hatte. Verstand sie beim Dictat ein Wort nicht oder unrichtig, so wurde es ihr aufgeschrieben, und sie musste es vorlesen, was auch jedesmal richtig erfolgte. Formittelst solcher Aufzeichnungen konnte ihr in späteren Sitzungen nachgewiesen werden, dass sie das Wort oder den Satz, der ihr augenblicklich Schwierigkeiten machte, bereits vor einigen Tagen anstandslos verstanden und nachgesprochen hatte. Die Besserung ging jetat vorwärts, und nach einem Aufenthalt von 14 Tagen in der Anstalt konnte das Kind als börend entlassen werden. Eine genaue qualitative und quantitative Bestimmung des Gehöres war nicht möglich, da die Aussagen des Kindes als nicht genügend glaubhaft erscheinen mussten.

Während wir zu Anfang annabmen, im Wesentlichen auf Grund der Anamnese, es handelte sich um complete Nerventaubheit im Gefolge von Typhns, mussten wir sehr bald doch einsehen, dass zum mindesten nicht unerhebliche Hörreste vorhanden waren. Deshalb glanbten wir, entweder eine Funetionsunfähigkeit eines Theiles der Leitungsbahnen oder eine solehe des sensoriellen centralen Apparates vor uns zu haben. Nun stellte sich aber heraus, dass von eigentlichen Hördefecten nicht gut die Rede war, dass vielmehr das Kind alles bören konnte, aber, sagen wir, zuweilen nicht hören wollte, resp. sich keine Mühe gab zu hören. Sollen wir diese Art schlechthin als Launenhaftigkeit bezeichnen, oder müssen wir sie doch als pathologisehen Vorgang auffassen. Wenn letzteres der Fall ist, so kommt nur die Erkranknng in Frage, in der gerade die Launenhaftigkeit in den versehiedensten Schattirungen eine grosse Rolle spielt, die Hysterie. Und eine hysterische Grundlage miussen wir bei unserer Kranken unbedingt annehmen. Eine Manifestation derselben war auch der merkwürdige Gang, den das Kind in den ersten Tagen seines Aufenthaltes in der Anstalt zeigte. Durch die rechtsseitige Kniegelenksankylose war derselbe nicht zu erklären, ebenso wenig waren Anhaltspunkte für ein Leiden der Centralorgane vorhanden. Und wenn wir nun die prompte Wirkung der Therapie berïck- 
sichtigen, die in energischer Faradisation der Unterschenkel bestand, und die nach zweimaliger Anwendung völlige Heilung des Zehenganges erreichte, so werden wir in der Beurtheilung der Motilitätsstörungen der unteren Extremitäten mit der Diagnose Hysterie keinen Feblgriff thun. Gerade der Umstand, dass wir es bei der Abnormität des Ganges, die ich vor einer Reihe von Jahren in gleicher Weise bei einem ziemlich gleich alten hysterischen Kinde schon einmal beobachtet habe, mit einem unzweifelhaft hysterischen Symptom zu thun hatten, rechtfertigt unsere Annabme, dass auch die Hörstöruug bei dem Kinde einzig und allein seiner Hysterie zur Last zu legen ist.

Von neueren Mitteln, welche im Verlanfe des Berichtsjahres in Anwendung kommen, mag hier nur das Schleich'sche Glutol erwähnt werden. Es stellt dieses Mittel, welches in grobkörniger Pulverform in. Handel kommt, bekanntlich eine Verbindung von Formalin und Gelatine dar und tritt erst durch Berührung mit der lebenden Zelle, welche nach Angabe des Erfinders eine Abspaltung des Formalins hervorruft, activ in Wirksamkeit. Es ist deshalb nothwendig nach Scbleich, dass reicblich lebensfähiges Gewebe mit dem Mittel in Berührung kommt; mortificirtes verhindert den Eintritt der Abspaltung des wirksamen Formalins. Wir wandten das Glutol hauptsächlich an bei Aufmeisselungen des Warzenfortsatzes in acuten Fälen, und zwar zu Anfang in der Weise, dass erst beim ersten Verbandwechsel (ca. 8 Tage nach der Operation) die bis dahin tamponirt gewesene Wundhöhle ausgiebig mit dem Mittel beschitttet wurde. Beim nächsten nnd den folgenden Verbandwechsel (für gewöbnlich in dreitägigem Turnus) fiel die geringe $W$ undsecretion auf. Die Wundränder sehen äusserst frisch aus ohne Reactionserscheinungen. In der Wundhöhle oder an der der Wunde zunächst liegenden Verbandsehicht findet sich eine homogene, graugelbliche, gallertige Masse, der Rest des gequollenen Glutols. Die Wundhöble hat sich ganz merklich verkleinert. Sie ist von guten Granulationen ausgekleidet, das Granulationspolster zeigt eine gleichmässige, glatte Oberfläche, welche an Schleimhaut erinnert. Von dem Grade der Secretion, die, wie gesagt, meist auffallend gering ist, bängt es $a b$, wie lange die Verbände liegen bleiben können. Wir haben bis 5 Tage gewartet, brauchten aber nie unter zweitägigen Wechsel herabzugehen. Infolge der Secret vermindernden und Zersetzung hemmenden.Wirkung des Mittels haben wir Eezem nur ganz selten und in minimaler Ausdehnung zu verzeichnen gehabt. 
Zuweilen beobachteten wir, dass nach längerer Anwendung des Glutols die Heilung anscheinend Stillstand machte. Die Wunde verkleinerte, sich nicht mehr zusehends, sah aber dabei frisch und ganz reactionslos aus. Wir haben dán das Mittel eine Zeitlang ansgesetzt und konnten uns tuberzeugen, dass es nach längerer Pause wieder in erwünschtem Maasse wirkte. Vielleicht ist gerade die Reactionslosigkeit der Wunde der Grund, dass die Heilungstendenz nachlässt. Es fehlt die Irritation, und damit fallen anch die Abwehrvorrichtungen, welche dem Organismus gegen solche Irritation za Gebote stehen, aus, in erster Linie die energische Zellproliferation, die ja doch schliesslich die Vernarbung vorzugsweise zu ubernehmen hat.

In einer Reihe von Fällen füllten wir gleich nach Beendigung der Operation die Wundhöhle mit Glutol aus. Zweimal beobachteten wir danach gleich am Operationstage und an den folgenden ein Ansteigen der Temperatur bis über $39^{\circ}$. Ein Wechsel des Verbandes und Entfernung des Glutols führte sofortige Entfieberung herbei. Es scheint doch also hier eine Retention pyrogener Stoffe stattgefunden zn haben, veranlasst, wie wir aus der schnellen Rückkehr der Temperatur zur Norm nach Entfernung des Glutols schliessen dürfen, durch das Mittel.

Dagegen baben wir aber auch bei der gleichen Anwendungsweise des Mittels zwei Heilungen zu verzeichnen, wie wir sie in so kurzer Zeit noch nie beobachtet haben. Im ersten Fall war es ein 6 jähriger Knabe, der nach Scharlach eine acute Mastoiditis acquirirt hatte. Die Erweichung war sehr ausgedehnt, die Knochenhöhle von der Grösse eines Fingergliedes. Beim ersten Verbandwechsel (nach einer Woche) war die Wunde auf die Hälfte verkleinert, Ohr trocken, nach weiteren 4 Tagen hatten die Granulationen das Niveau der Haut erreicht, und 22 Tage nach der Operation war die Wunde völlig gesehlossen.

Noch günstiger verlief die Heilung bei einem Weichensteller Anfangs der vierziger Jahre. Er hatte schon wochenlang seinen Angaben nach eine acute Mastoiditis gehabt. Operation am 16. Juni, Weite Ausdehnung der Erweichung, Spitze des Proc. entfernt. Um die oben erwähnten Retentionserscheinungen zu vermeiden, Drainagerohr in die Knochenhöhle, rings darum reichlich Glutol. Am 23. Juni 1. Verbandwechsel. Die ganze Wandhöhle bis anf den Drainagekanal mit Granulationen geschlossen. Ohr trocken. Sehr wenig gequollenes Glutol, das meiste resorbirt. 27. Juni 2. Verband, die Gelatine bildet gleichsam den Schorf 
auf der geschlossenen Wunde. 3. Juli. Die Wunde ist völlig vernarbt.

Die Heilung hat hier also nur 17 Tage erfordert. Wir hatten bei den zuletzt erwähnten Fällen den Eindruck, als ob das resorbirbare Material, die Gelatine, die Brücke bildete für die sich entwickelnden Granulation und ihren Zusammenschluss erleichterte. Es wïrden ja dies ähnliche Vorgänge sein, wie wir sie bei der Heilung unter dem feuchten Blutschorf oder bei dem Ersatz von Sehnenstücken durch resorbirbares Material sich abspielen sehen.

Warnen möchten wir vor Anwendung des Glutols in der Paukenhöhle. Wir hatten es einige Male bei Totalverlust des Trommelfelles versucht, durch Insufflation geringer Mengen des Pulvers die Secretion zu beeinflussen. Die Quellbarkeit des Mittels war aber eine derartig hochgradige, dass sie zu unliebsamen Störungen fuihrte. Es entstand ein mehr oder minder schmerzhafter Druck, der uns veranlasste, von weiterer Anwendung des Mittels in der Paukenböhle Abstand zu nehmen. 